\title{
Diffuse-interface approximations of osmosis free boundary problems
}

\author{
Andreas Rätz \\ Preprint 2015-04 \\ June 2015
}




\title{
DIFFUSE-INTERFACE APPROXIMATIONS OF OSMOSIS FREE BOUNDARY PROBLEMS
}

\author{
ANDREAS RÄTZ
}

\begin{abstract}
Free boundary problems based on mass conservation and surface tension with application in osmotic swelling are the topic of this contribution. We introduce new phase-field approximations of such models, in order to numerically investigate properties of the solutions. Formal justification of the proposed approximations is provided by matched asymptotic expansions supported by numerical tests reproducing the convergence for shrinking interface thickness.
\end{abstract}

\section{INTRODUCTION}

In the present paper, we consider a mathematical model for osmosis, i.e. we are interested in a membrane separating a region of a fluid with a higher solute concentration from a region with a lower concentration of a solute dissolved in the fluid. The membrane is called semipermeable, if it is permeable to the solvent but impermeable to the solute. A tendency to equal solute concentrations on both sides a semipermeable membrane can lead to a flux of the solvent through the membrane. Such osmotic phenomena play an important role in biological cells, since biological membranes are semipermeable.

1.1. A one-phase osmosis model. For the mathematical modeling of the above situation, we let $\Omega_{+}=\Omega_{+}(t) \subset \mathbb{R}^{n}, t \in[0, T]$, an open bounded time dependent domain bounded by a smooth closed hypersurface $\Gamma(t):=\partial \Omega_{+}(t)$ for all times $t$ under consideration. While $\Gamma$ represents the membrane, the inner of the membrane (in biological applications the cell) is given by $\Omega_{+}$. We denote the outer normal to $\Gamma$ by $\nu$ and define the mean curvature $H=\sum_{i=1}^{n-1} \kappa_{i}$ with principal curvatures $\kappa_{i}, i=1, \ldots, n-1$. Note that we use the sign convention that $H>0$ holds for $\Omega_{+}$convex. Furthermore, we consider the normal velocity $v$ of the interface. For the solute concentration $u(\cdot, t): \overline{\Omega_{+}(t)} \rightarrow \mathbb{R}, t \in[0, T]$, we study the one-phase free boundary model

$$
\begin{aligned}
\partial_{t} u-D_{+} \Delta u & =0 & & \text { in } \Omega_{+}, \\
-D_{+} \nabla u \cdot \nu-u v & =0 & & \text { on } \Gamma, \\
v & =-\alpha H+\beta u & & \text { on } \Gamma
\end{aligned}
$$

with diffusion coefficient $D_{+}$and positive parameters $\alpha$ and $\beta$ related to surface tension and osmotic pressure, respectively. Moreover, we assume suitable initial conditions $\Omega_{+}(0)=$

Date: May 28, 2015.

2010 Mathematics Subject Classification. 35R35,35K65,65N30.

Key words and phrases. free boundary problem, osmosis, phase-field model, diffuse interface, finite elements. 
$\Omega_{+, 0}$ and $u(\cdot, 0)=u_{0}$. From $(1.1)-(1.2)$ one obtains the conservation law

$$
\begin{aligned}
\frac{\mathrm{d}}{\mathrm{d} t} \int_{\Omega_{+}(t)} u(x, t) \mathrm{d} x & =\int_{\Gamma(t)} u(x, t) v(x, t) \mathrm{d} \sigma(x)+\int_{\Omega_{+}(t)} \partial_{t} u(x, t) \mathrm{d} x \\
& =\int_{\Gamma(t)} u(x, t) v(x, t) \mathrm{d} \sigma(x)+\int_{\Omega_{+}(t)} D_{+} \Delta u(x, t) \mathrm{d} x \\
& =\int_{\Gamma(t)} u(x, t) v(x, t) \mathrm{d} \sigma(x)+\int_{\Gamma(t)} D_{+} \nabla u(x, t) \cdot \nu(x, t) \mathrm{d} \sigma(x)=0 .
\end{aligned}
$$

The geometric evolution law (1.3) represents the competition between the surface tension and the osmotic force, where the first tends to shrink the membrane, while the latter tends to expand the membrane. The one-phase osmosis model $(1.1)-(1.3)$ corresponds to the case that the membrane $\Gamma$ and its inner $\Omega_{+}$are surrounded by the solvent with vanishing solute concentration. In this way it can be interpreted as a special case of the two-phase model described in the subsequent section 1.2 . Note that the free boundary problem (1.1) $-(1.3)$ admits for a stationary solution with $u$ spatially constant and $\Gamma$ spherical, which will be used for numerical tests in section 4.

Recently, several results have been found for the above free boundary problem and related models. A one dimensional variant of (1.1) -1.3 has been analytically and numerically investigated by Frischmuth and Hänler [7], see also [26] for a related model. More recently, Pickard [22] introduced a model for cell swelling based on proportionality of free energy differences and water flux without taking surface tension into account. Numerical simulations are included in $[22$ for rotationally symmetric situations. Short-time existence results for (1.1) - 1.3 have been proved in 13. In 29, a variational formulation in a gradient flow framework has been investigated (see also [30]). For well-posedness and regularity results for a class of free boundary problems including (1.1)-(1.3) as a special case, we refer to [31. Extensions of the above model with incorporation of viscous motion of the fluid have been proposed in by Lippoth, Peletier and Prokert [12], where short-time existence of classical solutions for the resulting problem has been proved.

1.2. A two-phase osmosis model. In addition to the previous one-phase model we study a two-phase osmosis model 14 . Here we consider an open bounded domain $\Omega \subset \mathbb{R}^{n}$ and a time dependent smooth interface $\Gamma=\Gamma(t), t \in[0, T]$, separating $\Omega$ into two time dependent sub domains $\Omega_{ \pm}=\Omega_{ \pm}(t), \Gamma(t)=\partial \Omega_{+}(t), t \in[0, T]$, such that $\Gamma=\overline{\Omega_{+}(t)} \cap \overline{\Omega_{-}(t)}$ and $\bar{\Omega}=\overline{\Omega_{+}(t)} \cup \overline{\Omega_{-}(t)}$ hold for all times $t$. Then the two-phase osmosis free boundary problem with $u_{ \pm}(\cdot, t): \overline{\Omega_{ \pm}(t)} \rightarrow \mathbb{R}$ reads

$$
\begin{aligned}
\partial_{t} u_{ \pm}-D_{ \pm} \Delta u_{ \pm} & =0 & & \text { in } \Omega_{ \pm}, \\
D_{ \pm} \nabla u_{ \pm} \cdot \nu+u_{ \pm} v & =0 & & \text { on } \Gamma, \\
v & =-\alpha H+\beta[u] & & \text { on } \Gamma, \\
\partial_{\nu} u_{-} & =0 & & \text { on } \Sigma
\end{aligned}
$$

with $\Sigma:=\partial \Omega$ and initial conditions $\Omega_{ \pm}(0)=\Omega_{ \pm, 0}$ and $u_{ \pm}(\cdot, 0)=u_{ \pm, 0}$. Moreover, by $[u]:=\left.u_{+}\right|_{\Gamma}-\left.u_{-}\right|_{\Gamma}$ we denote the jump of the solute concentration across the interface. Note that choosing the initial conditions for $\Omega_{ \pm}$corresponds to choosing an initial interface 
$\Gamma(0)=\Gamma_{0}$. For the above two-phase model, the conservation law analogous to (1.4) reads

$$
\begin{aligned}
\frac{\mathrm{d}}{\mathrm{d} t}\left(\int_{\Omega_{+}} u_{+}+\int_{\Omega_{-}} u_{-}\right) & =\int_{\Gamma}\left(u_{+}-u_{-}\right) v+\int_{\Omega_{+}} \partial_{t} u_{+}+\int_{\Omega_{-}} \partial_{t} u_{-} \\
& =\int_{\Gamma}\left(u_{+}-u_{-}\right) v+\int_{\Omega_{+}} D_{+} \Delta u_{+}+\int_{\Omega_{-}} D_{-} \Delta u_{-} \\
& =\int_{\Gamma}\left(u_{+}-u_{-}\right) v+\int_{\Gamma}\left(D_{+} \nabla u_{+}-D_{-} \nabla u_{-}\right) \cdot \nu=0 .
\end{aligned}
$$

We would like to remark, that in the special case $u_{-}(x, t) \equiv 0$ the two-phase model (1.5)(1.8) reduces to the one-phase model (1.1)-1.3).

1.3. Outline of the paper. In this paper, we introduce phase-field approximations of both the one-phase and the two-phase model. In such a diffuse-interface approximation the interface is implicitly given by a phase-field function, which is obtained, roughly speaking, by smearing out the indicator function of $\Omega_{+}$on a short length $\mathcal{O}(\varepsilon)$ for some small parameter $\varepsilon>0$. The diffuse domain method [9, 10, 11 offers a powerful tool for the approximation of free boundary problems [23, 25] and coupled bulk-surface PDE-systems [27, 24]. It will be used here, in order to numerically simulate the one- and two-phase models previously introduced. Apart from the numerical investigations of a simplified version of the one-phase model in one space dimension in [7, this contribution is the first attempt to provide a systematic numerical treatment of the one- and two-phase models (1.1) -1.3 . and (1.5)-(1.8), respectively, in full generality.

In section 2 , we introduce the diffuse approximations of $(1.1)-(1.3)$ and $(1.5)-(1.8)$, respectively. These are related to a phase-field model for precipitation in porous media, recently proposed in [25]. The phase-field approximations are justified by matched asymptotic expansions formally showing convergence of the diffuse-interface models towards the sharp-interface counterparts as the width of the diffuse interface shrinks to zero (section 3).

For the phase-field approximations, we propose in section 4 finite element discretizations, which are semi implicit in time. These schemes are used in order to recover the convergence towards the original sharp-interface models in rotationally symmetric, quasi-stationary situations. Further numerical examples are included to provide more insight to the properties of the model and its solutions.

\section{Diffuse-Interface approximations}

For the numerical approximation of solutions of the one- and two-phase models (1.1) (1.3) and (1.5)-(1.7), respectively, we use a phase-field approach. The key ingredient behind this ansatz is the diffuse-domain [9, 10, 11] approximation, where boundary value problems are described in a phase-field context. For a closely related model for precipitation in porous media, recently a phase-field model has been proposed in 25]. As described in [25], this phase-field model has the same structure as the model introduced in [20]. Rigorous convergence proofs for the diffuse domain method approximating second order elliptic boundary value problems have been provided in [5]. Moreover, in [1] a diffuse domain approach of a linear coupled elliptic PDE system has been analyzed. Thereby, well-posedness of the diffuse approximation and convergence results for interface thickness tending to zero have been proved. 
2.1. One-phase model. In order to provide a diffuse-interface approximation of the onephase model (1.1)-(1.3), we choose a (simple computational) domain $\Omega \subset \mathbb{R}^{n}$ containing $\overline{\Omega_{+}(t)} \forall t \in[0, T]$. The idea in such diffuse-interface (or "phase-field") approximation is to smear out the indicator function of $\Omega_{+}$on a short length of order $\mathcal{O}(\varepsilon)$ for a small parameter $\varepsilon>0$. This approach is based on a Ginzburg-Landau free energy

$$
\mathcal{H}_{\varepsilon}(\varphi):=\int_{\Omega}\left(\frac{\varepsilon}{2}|\nabla \varphi|^{2}+\varepsilon^{-1} G(\varphi)\right)
$$

for the smeared out function $\varphi$, where

$$
G=G(\varphi):=18 \varphi^{2}(1-\varphi)^{2}
$$

denotes a double well potential normalized such that

$$
\int_{0}^{1} \sqrt{2 G(\varphi)} \mathrm{d} \varphi=1
$$

holds. By the celebrated Modica-Mortola theorem (2.1) Gamma-converges with respect to $L^{1}$ to the perimeter functional [18, 17]

$$
\mathcal{H}(\Gamma)=\mathcal{H}^{n-1}(\Gamma)
$$

Taking only normal variations of the surface $\Gamma$ into account the functional derivative of the perimeter functional reads $\frac{\delta \mathcal{H}}{\delta \Gamma}=-H$. Thus, a natural diffuse-interface counterpart of mean curvature is given by

$$
H_{\varepsilon}:=\frac{\delta \mathcal{H}_{\varepsilon}}{\delta \varphi}=-\varepsilon \Delta \varphi+\varepsilon^{-1} G^{\prime}(\varphi)
$$

With this ansatz in mind we end up with a diffuse-interface approximation

$$
\begin{aligned}
\partial_{t}(\varphi u)-D_{+} \nabla \cdot(\varphi \nabla u)=0 & \text { in } \Omega \times(0, T), \\
\varepsilon \partial_{t} \varphi=\alpha\left(\varepsilon \Delta \varphi-\varepsilon^{-1} G^{\prime}(\varphi)\right)+\beta g(\varphi) u & \text { in } \Omega \times(0, T)
\end{aligned}
$$

of the sharp-interface one-phase osmosis model (1.1) $-(1.3)$, where in $(2.3)$ the phase-field function $\varphi$ has been used in order to restrict diffusion to the part of $\Omega$ which is inside the interface, see [11]. In addition, one can use

$$
g(\varphi):=\sqrt{2 G(\varphi)}
$$

or

$$
g(\varphi):=30 \varphi^{2}(1-\varphi)^{2}
$$

where the latter is our choice for the numerical simulations in section 4 . Note that if we neglect the last term on the right hand side of 2.4), it coincides with the Allen-Cahn equation [3], which is a standard phase-field approximation of mean curvature flow, see [19, 6, 8] for rigorous convergence results. The asymptotic analysis will show that (2.3) not only includes a diffuse-interface description of the diffusion equation (1.1) but also encodes the boundary condition $(1.2)$.

The diffuse conservation law corresponding to (1.4) reads

$$
\frac{\mathrm{d}}{\mathrm{d} t} \int_{\Omega} \varphi(x, t) u(x, t) \mathrm{d} x=\int_{\Omega} \partial_{t}(\varphi(x, t) u(x, t)) \mathrm{d} x=\int_{\Omega} D_{+} \nabla \cdot(\varphi(x, t) \nabla u(x, t))=0,
$$

where we have assumed no flux boundary condition $\varphi \nabla u \cdot \nu_{\partial \Omega}=0$ on $\partial \Omega, \nu_{\partial \Omega}$ denoting the outer normal to $\partial \Omega$. Moreover, we assume $\nabla \varphi \cdot \nu_{\partial \Omega}=0$ on $\partial \Omega$ and initial conditions for 
$u$ and $\varphi$ in $\Omega$ which correspond to initial conditions of the original sharp-interface model (1.1)-(1.3).

2.2. Two-phase model. For the diffuse-interface approximation of the two-phase sharpinterface model (1.5)-(1.7), we propose the natural extension

$$
\begin{aligned}
\partial_{t}\left(\varphi u_{+}\right)-D_{+} \nabla \cdot\left(\varphi \nabla u_{+}\right)=0 & \text { in } \Omega \times(0, T), \\
\partial_{t}\left((1-\varphi) u_{-}\right)-D_{-} \nabla \cdot\left((1-\varphi) \nabla u_{-}\right)=0 & \text { in } \Omega \times(0, T), \\
\varepsilon \partial_{t} \varphi=\alpha\left(\varepsilon \Delta \varphi-\varepsilon^{-1} G^{\prime}(\varphi)\right)+\beta g(\varphi)\left(u_{+}-u_{-}\right) & \text {in } \Omega \times(0, T),
\end{aligned}
$$

of the above diffuse one-phase model, where $\Omega=\overline{\Omega_{+}(t)} \cup \overline{\Omega_{-}(t)}$. In $(2.7)$, the function $1-\varphi$ is used in order to restrict the diffusion to the part outside the interface. Again, we choose initial conditions related to the ones of the sharp-interface model as well as boundary conditions for $\varphi$ and $u_{ \pm}$on $\partial \Omega$. We remark that this approach is similar to the proposed in [23], where a related phase-field model has been used in order to approximate a Stefan-like free boundary ("Burton-Cabrera-Frank") step flow model in epitaxial growth.

The diffuse-interface counterpart of the conservation law (1.9) now reads

$$
\frac{\mathrm{d}}{\mathrm{d} t} \int_{\Omega}\left(\varphi(x, t) u_{+}(x, t)+(1-\varphi(x, t)) u_{+}(x, t)\right) \mathrm{d} x=0
$$

similar to the one-phase case and again assuming no-flux boundary conditions $\varphi \nabla u_{+} \cdot \nu_{\partial \Omega}=$ 0 and $(1-\varphi) \nabla u_{-} \cdot \nu_{\partial \Omega}=0$ on $\partial \Omega$. As for the one-phase model, we will assume $\nabla \varphi \cdot \nu_{\partial \Omega}=0$ on $\partial \Omega$ for our numerical simulations.

\section{Matched ASymptotic EXPAnsions}

In this section, we provide matched asymptotic expansions (see e.g. 21]) for the approximation of the above two-phase-model by the diffuse-interface model (2.6)-(2.8). In order to achieve more rigorous results, one would have to apply techniques from [19, 6, 8] for the Allen-Cahn equation or [2] for the Cahn-Hilliard equation.

We closely follow the asymptotic expansions in [11, 23] and apply the setup in [23]. The outer solution is an approximation to the solution in the solvent, the inner solution zooms in at the interface. We make the following ansatz for the outer expansions:

$$
\begin{gathered}
u_{+}=u_{+, 0}+\mathcal{O}(\varepsilon), \\
u_{-}=u_{-, 0}+\mathcal{O}(\varepsilon), \\
\varphi=\varphi_{0}+\mathcal{O}(\varepsilon) .
\end{gathered}
$$

We consider a smeared-out interface connecting the inner of a membrane where $\varphi \approx 0$ to the outer of a membrane where $\varphi \approx 1$. Let $\Gamma$ denote the interface $\left\{\varphi=\frac{1}{2}\right\}$. Let $\nu$ denote the outer normal of $\Gamma, H$ its mean curvature, $v$ its normal velocity. We further introduce new coordinates in a neighborhood of the interface. To this end, we consider a (local) parametric representation $X: S \times[0, T] \rightarrow \Gamma \subset \mathbb{R}^{d}$ of $\Gamma$, where $S$ is an open subset of $\mathbb{R}^{d-1}$. Moreover, $r=r(x, t)$ is defined as the signed distance of $x$ from $\Gamma$ being positive outside $\Gamma$. We assume that in a sufficiently small neighborhood of $\Gamma$ one can write $x=X(s, t)+r(x, t) \nu(s, t)$. Now one transforms $u_{+}, u_{-}$and $\varphi$ to the new coordinate 
system:

$$
\begin{aligned}
\hat{u}_{+}(r, s, t) & :=u_{+}(X(s, t)+r \nu(s, t), t), \\
\hat{u}_{-}(r, s, t) & :=u_{-}(X(s, t)+r \nu(s, t), t), \\
\hat{\varphi}(r, s, t) & :=\varphi(X(s, t)+r \nu(s, t)) .
\end{aligned}
$$

Furthermore a stretched variable is introduced $z:=\frac{r}{\varepsilon}$, and one defines

$$
\begin{aligned}
U_{+}(z, s, t) & :=\hat{u}_{+}(r, s, t), \\
U_{-}(z, s, t) & :=\hat{u}_{-}(r, s, t), \\
\Phi(z, s, t) & :=\hat{\varphi}(r, s, t) .
\end{aligned}
$$

We assume outer expansions

$$
\begin{aligned}
& \hat{u}_{+}(r, s, t)=\hat{u}_{+, 0}(r, s, t)+\mathcal{O}(\varepsilon), \\
& \hat{u}_{-}(r, s, t)=\hat{u}_{-, 0}(r, s, t)+\mathcal{O}(\varepsilon), \\
& \hat{\varphi}(r, s, t)=\hat{\varphi}_{0}(r, s, t)+\mathcal{O}(\varepsilon)
\end{aligned}
$$

and inner expansions

$$
\begin{aligned}
& U_{+}(z, s, t)=U_{+, 0}(z, s, t)+\varepsilon U_{+, 1}(z, s, t)+\varepsilon^{2} U_{+, 2}(z, s, t)+\mathcal{O}\left(\varepsilon^{3}\right), \\
& U_{-}(z, s, t)=U_{-, 0}(z, s, t)+\varepsilon U_{-, 1}(z, s, t)+\varepsilon^{2} U_{-, 2}(z, s, t)+\mathcal{O}\left(\varepsilon^{3}\right), \\
& \Phi(z, s, t)=\Phi_{0}(z, s, t)+\varepsilon \Phi_{1}(z, s, t)+\mathcal{O}\left(\varepsilon^{2}\right)
\end{aligned}
$$

With this ansatz, we have

$$
\begin{aligned}
& \partial_{t} u_{+}=-\varepsilon^{-1} v \partial_{z} U_{+}+O(1), \\
& \nabla u_{+}=\varepsilon^{-1} \partial_{z} U_{+} \nu+\sum_{i, j=1}^{n-1} g^{i j} \partial_{s_{i}} U_{+} \partial_{s_{j}} X+\mathcal{O}(\varepsilon), \\
& \Delta u_{+}=\varepsilon^{-2} \partial_{z}^{2} U_{+}+\epsilon^{-1} H \partial_{z} U_{+}+\Delta_{\Gamma} U_{+}+\mathcal{O}(\varepsilon),
\end{aligned}
$$

where $g_{i j}:=\partial_{s_{i}} X \cdot \partial_{s_{j}} X$ and $\left(g^{i j}\right):=\left(g_{i j}\right)^{-1}$. Corresponding relations hold for $u_{-}, \varphi$ and $U_{-}, \Phi$, respectively. Furthermore, we use matching conditions on inner and outer solutions

$$
\begin{aligned}
\lim _{z \rightarrow \pm \infty} U_{+, 0} & =\lim _{r \rightarrow \pm 0} \hat{u}_{+, 0}, & \lim _{z \rightarrow \pm \infty} \partial_{z} U_{+, 1} & =\lim _{r \rightarrow \pm 0} \partial_{r} \hat{u}_{+, 0}=\lim _{r \rightarrow \pm 0} \partial_{\nu} u_{+, 0}, \\
\lim _{z \rightarrow \pm \infty} U_{-, 0} & =\lim _{r \rightarrow \pm 0} \hat{u}_{-, 0}, & \lim _{z \rightarrow \pm \infty} \partial_{z} U_{-, 1} & =\lim _{r \rightarrow \pm 0} \partial_{r} \hat{u}_{-, 0}=\lim _{r \rightarrow \pm 0} \partial_{\nu} u_{-, 0}, \\
\lim _{z \rightarrow \pm \infty} \Phi_{0} & =\lim _{r \rightarrow \pm 0} \hat{\varphi}_{0} . & &
\end{aligned}
$$

3.1. Outer Expansions. The outer expansions to order $O\left(\varepsilon^{-1}\right)$ yield in $(2.8)$

$$
G^{\prime}\left(\varphi_{0}\right)=0 \quad \Longrightarrow \quad \varphi_{0} \in\{0,1\}
$$

where we have excluded the unstable solution $\varphi_{0}=\frac{1}{2}$. From (3.13) we get in (2.6) and (2.7) to $O\left(\varepsilon^{0}\right)$

$$
\begin{aligned}
& \partial_{t}\left(u_{-, 0}\right)=D_{-} \Delta u_{-, 0} \quad \text { where } \quad \varphi_{0}=0, \\
& \partial_{t}\left(u_{+, 0}\right)=D_{+} \Delta u_{+, 0} \quad \text { where } \quad \varphi_{0}=1,
\end{aligned}
$$

i.e. diffusion equations 1.5 for $u_{-, 0}$ and $u_{+, 0}$ outside and inside the membrane, respectively. 
3.2. Inner Expansions. The inner expansions in $(2.8)$ lead to

$$
\partial_{z}^{2} \Phi_{0}-G^{\prime}\left(\Phi_{0}\right)=0
$$

to order $O\left(\varepsilon^{-1}\right)$. From (3.12), we get to leading order $O\left(\varepsilon^{-2}\right)$ in $(2.6), 2.7$

$$
\partial_{z} U_{-, 0}=\partial_{z} U_{+, 0}=0,
$$

and to order $O\left(\varepsilon^{-1}\right)$

$$
\begin{aligned}
-v \partial_{z}\left(\Phi_{0} U_{+, 0}\right) & =D_{+} \partial_{z}\left(\Phi_{0} \partial_{z} U_{+, 1}\right) \\
-v \partial_{z}\left(\left(1-\Phi_{0}\right) U_{-, 0}\right) & =D_{-} \partial_{z}\left(\left(1-\Phi_{0}\right) \partial_{z} U_{-, 1}\right) .
\end{aligned}
$$

Integration leads to

$$
\begin{aligned}
v \lim _{z \rightarrow-\infty} U_{+, 0} & =-D_{+} \lim _{z \rightarrow-\infty} \partial_{z} U_{+, 1}, \\
-v \lim _{z \rightarrow \infty} U_{-, 0} & =D_{-} \lim _{z \rightarrow \infty} \partial_{z} U_{-, 1},
\end{aligned}
$$

and hence, by matching conditions, $u_{-, 0}$ and $u_{+, 0}$ fulfill the boundary conditions (1.6).

Going back to (2.8), we obtain in $O\left(\varepsilon^{0}\right)$

$$
-v \partial_{z} \Phi_{0}=\alpha\left(\partial_{z}^{2} \Phi_{1}-G^{\prime \prime}\left(\Phi_{0}\right) \Phi_{1}+H \partial_{z} \Phi_{0}\right)+\beta g\left(\Phi_{0}\right)\left(U_{+, 0}-U_{-, 0}\right),
$$

which gives by testing $O\left(\varepsilon^{0}\right)$ with $\partial_{z} \Phi_{0}$ the evolution law

$$
v=-\alpha H+\beta\left(\lim _{r \rightarrow-0} u_{+, 0}-\lim _{r \rightarrow+0} u_{-, 0}\right)
$$

which is equivalent to 1.7$)$. Thus, we have formally shown the convergence of the phasefield model (2.6)-(2.8) towards its sharp-interface counterpart (1.5)-1.7) for diffuse interface thickness tending to zero.

\section{Numerical Results}

In this section, we present numerical results for the diffuse-interface approximations (2.3) - 2.4) and (2.6)-(2.8) for the one-phase and two-phase sharp-interface counterparts, respectively. Thereby, we use a spatial finite element discretization combined with a semiimplicit discretization in time.

In section 4.1 we describe the discretization of the phase-field approximations. Moreover, in sections 4.2 and 4.3 we present numerical results for the approximation of the one-phase and two-phase models. Thereby, we compare the numerical results with analytic solutions for rotationally symmetric quasi-stationary cases in dimension $n=2$, and we further provide simulation results showing properties of the models.

4.1. Semi-implicit finite element discretization. Here, we only describe the numerical treatment of the diffuse-interface one-phase system (2.3)-(2.4). The discretization of $(2.6)-$ (2.8) is then a straight forward generalization. 
We introduce time instants $\tau_{m}, m=1, \ldots, M_{T}$. First we provide a time discrete weak formulation of (2.3)-(2.4)

$$
\begin{aligned}
\text { (4.1) } & \int_{\Omega} \frac{\varphi^{(m)} u^{(m+1)}}{\tau_{m+1}} \psi+\int_{\Omega} \frac{\varphi^{(m+1)} u^{(m)}}{\tau_{m+1}} \psi+D_{+} \int_{\Omega} b\left(\varphi^{(m)}\right) \nabla u^{(m+1)} \cdot \nabla \psi \\
& =2 \int_{\Omega} \frac{\varphi^{(m)} u^{(m)}}{\tau_{m+1}} \psi \\
\text { (4.2) } \varepsilon & \int_{\Omega} \frac{\varphi^{(m+1)}}{\tau_{m+1}} \eta+\alpha \varepsilon \int_{\Omega} \nabla \varphi^{(m+1)} \cdot \nabla \eta-\beta \int_{\Omega} g\left(\varphi^{(m)}\right) u^{(m+1)} \eta+\frac{\alpha}{\varepsilon} \int_{\Omega} G^{\prime \prime}\left(\varphi^{(m)}\right) \varphi^{(m+1)} \eta \\
& =\varepsilon \int_{\Omega} \frac{\varphi^{(m)}}{\tau_{m+1}} \eta+\frac{\alpha}{\varepsilon} \int_{\Omega}\left(G^{\prime \prime}\left(\varphi^{(m)}\right) \varphi^{(m)}-G^{\prime}\left(\varphi^{(m)}\right)\right) \eta
\end{aligned}
$$

for all $\psi, \eta \in H^{1}(\Omega)$. Thereby, we have used $b(s):=\delta+s_{+}, s \in \mathbb{R}$, for a small regularizing parameter $\delta>0$ and the positive part $s_{+}:=\max (0, s)$ of $s$, in order to regularize the otherwise degenerate equation. To discretize in space, we use linear finite elements and a simple strategy of adaptive mesh refinement and coarsening similar to the one described in [4] based on the values of the discrete phase-field function. The resulting linear system of PDE's is solved by a stabilized bi-conjugate gradient (BiCGStab) method. This numerical scheme is implemented in the adaptive FEM toolbox AMDiS [28].

4.2. Numerical examples for the one-phase model. We apply the above numerical scheme for the phase-field approximation of the one-phase model and compare numerical and analytic solutions. Moreover, we investigate the influence of the concentration on the membrane shape and vice versa.

4.2.1. Approaching stationary solutions: the one-phase model. For given initial conditions $u_{0}$ and $\Omega_{+, 0}=B_{R_{0}}(0)$ with initial radius $R_{0}$, we consider as a first benchmark the stationary solutions

$$
\Omega_{\infty}=B_{R_{\infty}}(0), \quad u_{\infty}=\frac{\pi \alpha^{2}}{M \beta^{2}}
$$

of the one-phase model $1.1-1.3$ for $n=2$, where $R_{\infty}=\frac{M \beta}{\pi \alpha}$ and

$$
M:=\int_{\Omega_{+}, 0} u_{0}=\pi R_{\infty}^{2} u_{\infty}
$$

denotes the (conserved) total mass. We apply the above numerical scheme of the phasefield approximation (2.3) -2.4 with $\Omega=(-2,2)^{2}$ and approximation parameter $\delta=10^{-5}$, $\varepsilon=0.1$. Moreover, we apply a diffuse-interface approximation $\varphi(\cdot, 0)$ of $\chi_{\Omega_{0}}$. For the sharp-interface model, we choose $u_{0}=\frac{1}{2}, D_{+}=1, \beta=1$. In Fig. 1 we see plots of the diffuse area

$$
A_{\varepsilon}(t):=\int_{\Omega} \varphi(\cdot, t) \approx\left|\Omega_{+}(t)\right|
$$

versus time $t$ for two different scenarios: the approximation of

- a growth scenario $\left(\alpha=\frac{1}{8}\right.$ and $\left.R_{0}=\frac{1}{2}\right)$ (left)

- a shrinkage scenario $\left(\alpha=1\right.$ and $\left.R_{0}=1\right)$ (right)

each towards a stationary solution with area $\pi R_{\infty}^{2}$. 

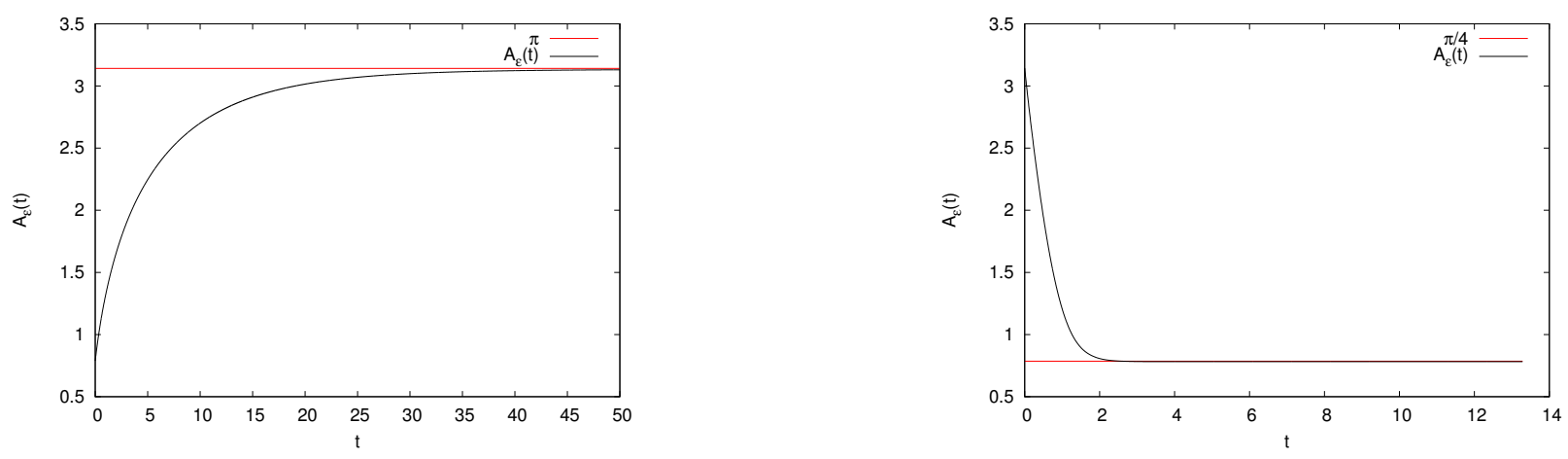

FiguRE 1. Diffuse area $A_{\varepsilon}$ versus time $t$ for $\alpha=\frac{1}{8}$ and $R_{0}=\frac{1}{2}$ (left) and for $\alpha=1$ and $R_{0}=1$ (right) compared with area corresponding to analytic stationary solution in 4.3 .

4.2.2. Comparison with analytic results. For a quantitative comparison of numerical results with an analytic solution, we consider the following rotationally symmetric and quasistationary situation. In the formal limit $D_{+} \rightarrow \infty$ one obtains from the one-phase system (1.1) - 1.3) the quasi-stationary system

$$
\begin{aligned}
-\Delta u & =0 & & \text { in } \Omega_{+}(t), t>0, \\
-\nabla u \cdot \nu & =0 & & \text { on } \Gamma(t), t>0, \\
v & =-\alpha H+\beta u & & \text { on } \Gamma(t), t>0 .
\end{aligned}
$$

Thus, $u$ is spatially constant, $u(x, t) \equiv: \hat{u}(t)$, where $\hat{u}$ is given by mass conservation

$$
\hat{u}(t)\left|\Omega_{+}(t)\right|=\int_{\Omega_{+}(t)} u(x, t) \mathrm{d} x=\int_{\Omega_{+}(0)} u(x, 0) \mathrm{d} x=\int_{\Omega_{0}} u_{0}(x) \mathrm{d} x .
$$

For our numerical tests we assume $\Omega_{+}(t)=B_{R(t)}(0) \forall t$ and constant initial data $u_{0}$. Then for $n=2$ one obtains from $4.5-4.7$ the ODE

$$
\dot{R}=-\frac{\alpha}{R}+\frac{\beta u_{0} R_{0}^{2}}{R^{2}}
$$

with initial radius $R(0)=R_{0}$, which we solve numerically with Maple ${ }^{\mathrm{TM}}[15$, in order to investigate the approximation of the analytic solution for decreasing values of $\varepsilon$. In Fig. 2 we compare the perimeter $L(t)=2 \pi R(t)$ corresponding to $(4.8)$ with the diffuse-interface counterparts

$$
L_{\varepsilon}(t)=\int_{\Omega}\left(\frac{\varepsilon}{2}|\nabla \varphi(\cdot, t)|^{2}+\varepsilon^{-1} G(\varphi(\cdot, t))\right)
$$

obtained from simulation results of the discretization of $(2.3)$, (2.4) for different $\varepsilon$ values and for $\Omega=(-2,2)^{2}, D_{+}=100, \delta=10^{-5}$. Moreover, we see the stationary perimeter $2 \pi$ due to the parameters $\alpha=\frac{1}{8}, u_{0}=\frac{1}{2}, \beta=1$ and $R_{0}=\frac{1}{2}$. One observes the approximation of the analytic solution for decreasing $\varepsilon$ as predicted by the asymptotic analysis in section 3.

As a further validation of the numerical scheme, we consider the diffuse mass conservation law (see also 2.5)

$$
M_{+}(t):=\int_{\Omega} \varphi(x, t) u(x, t) \mathrm{d} x \equiv M_{+}(0) .
$$




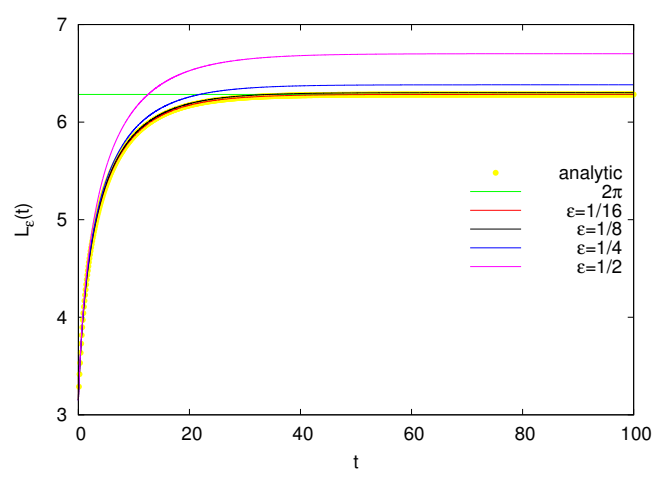

FiguRE 2. Comparison of the analytic perimeter $L=L(t)=2 \pi R(t)$ with $R=R(t)$ given by the ODE (4.8) with initial condition $R_{0}=\frac{1}{2}$ with diffuse length $L_{\varepsilon}$ versus time $t$ for various values of $\varepsilon$.

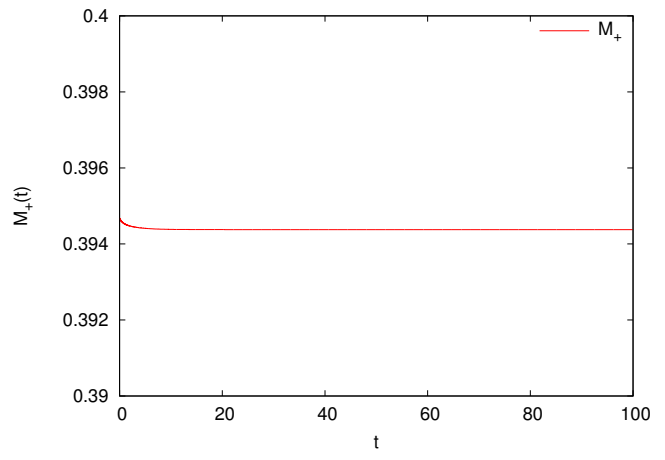

FIGURE 3. Discrete mass versus time for $\varepsilon=\frac{1}{8}$.

In Fig. 3 we see the discrete mass versus time for the case $\varepsilon=\frac{1}{8}$ of the previous example, with $\varphi$ and $u$ replaced by discrete solutions $\varphi_{h}$ and $u_{h}$ in 4.9 . The results show a reasonable approximation of the conservation law 4.9 .

4.2.3. Further numerical results. In the following we show further simulation results of the diffuse-interface approximation (2.3)-(2.4) of the one-phase osmosis model (1.1)-(1.3), where we have studied the influence of various initial conditions. Moreover, we have used $\varepsilon=0.1$ and $\delta=10^{-5}$. For $n=2$ we have triangulated $\Omega=(-10,10)^{2}$, and for $n=3$ we have considered $\Omega=(-5,5)^{3}$.

I: Ellipse as initial membrane shape. As initial conditions we assume an ellipse for $\Gamma_{0}$ and a constant value for $u_{0}$ :

$$
\Omega_{+}(0)=\left\{(x, y) \in \mathbb{R}^{2}: \frac{x^{2}}{a^{2}}+a^{2} y^{2}<1\right\}, u_{0} \equiv 1 .
$$

Further parameters used for this example are

$$
D_{+}=1 ; \quad \alpha=0.2 ; \quad \beta=1 ; \quad a=5 .
$$

Results of this situation can be seen in Fig. 4, where the conserved quantity $\varphi_{h} u_{h}$ is displayed in a contour plot with additional plot of the level line $\left\{\varphi_{h}=\frac{1}{2}\right\}$. We see that the initially constant discrete concentration becomes non-constant and the interface evolves towards a circle. 

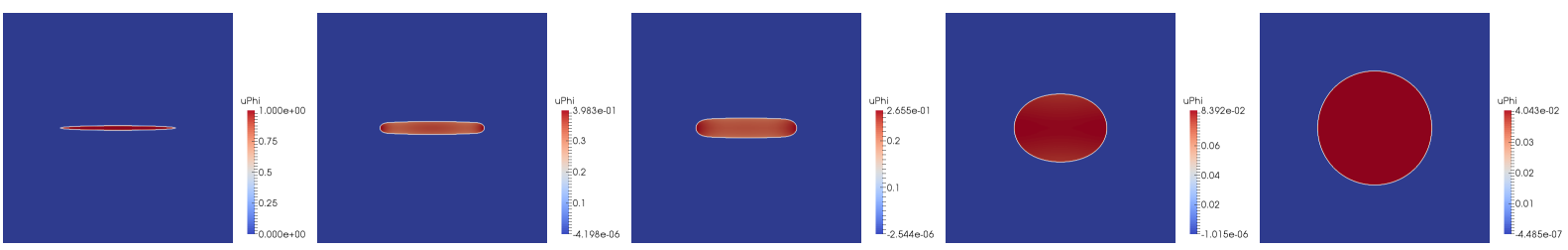

FIGURE 4. Elliptic initial membrane shape. Discrete concentration $\varphi_{h} u_{h}$ and level set $\left\{\varphi_{h}=\frac{1}{2}\right\}$ at times $t=0, t \approx 0.76, t \approx 1.93, t \approx 25.12$ and $t \approx 1000$.
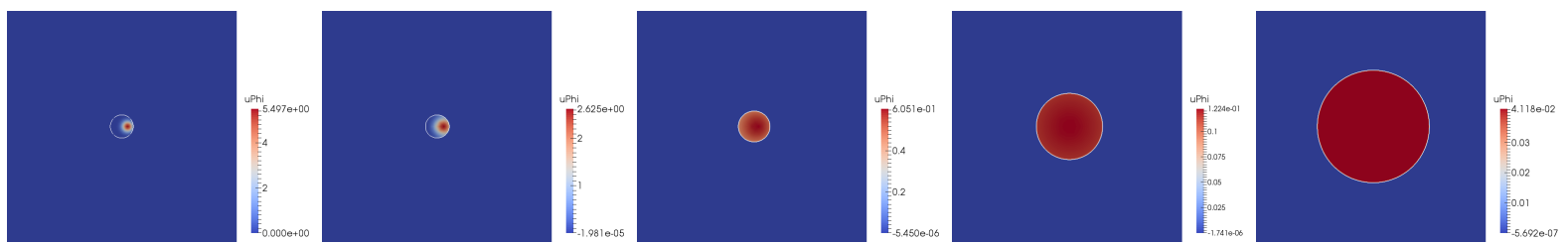

FIGURE 5. Locally increased initial solute concentration and circular initial membrane shape. Discrete concentration $\varphi_{h} u_{h}$ and level set $\left\{\varphi_{h}=\frac{1}{2}\right\}$ at times $t=0, t \approx 0.06$, $t \approx 0.87, t \approx 16.29$ and $t \approx 50$.

II: Locally increased initial solute concentration. In a second example we consider locally increased initial solute concentration and a circular initial membrane shape:

$$
\Omega_{+}(0)=B_{1}(0), u_{0}(x, y)=B\left(a_{u}\right) \exp \left(-a_{u}\left(\left(x-x_{0}\right)^{2}+\left(y-y_{0}\right)^{2}\right)\right)
$$

for $(x, y) \in \Omega, x_{0}=\frac{1}{2}, y_{0}=0, a_{u}=5$ and $B\left(a_{u}\right)$ is chosen such that $M=\pi$ holds. In addition, we use

$$
D_{+}=1 ; \quad \alpha=0.2 ; \quad \beta=1 .
$$

In Fig. 5, one can see plots of $\varphi_{h} u_{h}$ at different times showing only little influence of the solute concentration on the membrane shape.

\section{III: Perturbation of circular membrane shape and locally increased solute con- centration.}

2d: Next we use a perturbation of a circular initial membrane shape and the locally increased initial solute concentration from the previous example:

$$
\Omega_{+}(0)=\{(r \cos \theta, r \sin \theta): 0 \leq r<1+A \cos (m \theta), 0 \leq \theta \leq 2 \pi\}
$$

with $m=6, A=0.6$ and the initial function $u_{0}$ from II with $a_{u}=20, x_{0}=1.8$, $y_{0}=0$. Moreover, the parameters

$$
D_{+}=0.01 ; \quad \alpha=0.2 ; \quad \beta=1
$$

have been used. Results for the discrete conserved concentration $\varphi_{h} u_{h}$ in this example are shown in Fig. 6. According to the bump in the initial condition, one observes asymmetric intermediate shapes as well as lateral shift of the membrane. Another example is given in Fig. 7, where we consider an initial function $u_{0}$ with two local maxima.

3d: Similar to the 2d-case, we consider a perturbation of a sphere

$$
\Gamma(0)=\left\{R_{\psi}\left(\begin{array}{c}
\left(1+A_{\theta} \cos \left(k_{\theta} \theta\right)\right) \cos (\psi) \cos (\theta) \\
\left(1+A_{\theta} \cos \left(k_{\theta} \theta\right)\right) \cos (\psi) \sin (\theta) \\
\sin (\psi)
\end{array}\right): 0 \leq \theta \leq 2 \pi,-\frac{\pi}{2} \leq \psi \leq \frac{\pi}{2}\right\}
$$



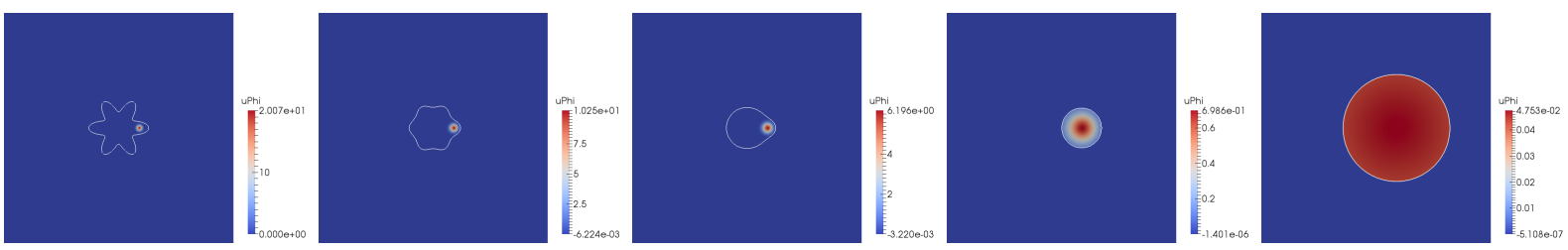

FiguRE 6. Perturbation of a circular initial membrane shape and the locally increased initial solute concentration. Discrete concentration $\varphi_{h} u_{h}$ and level set $\left\{\varphi_{h}=\frac{1}{2}\right\}$ at times $t=0, t \approx 0.986, t \approx 2.495, t \approx 35.255$ and $t \approx 1501.151$.
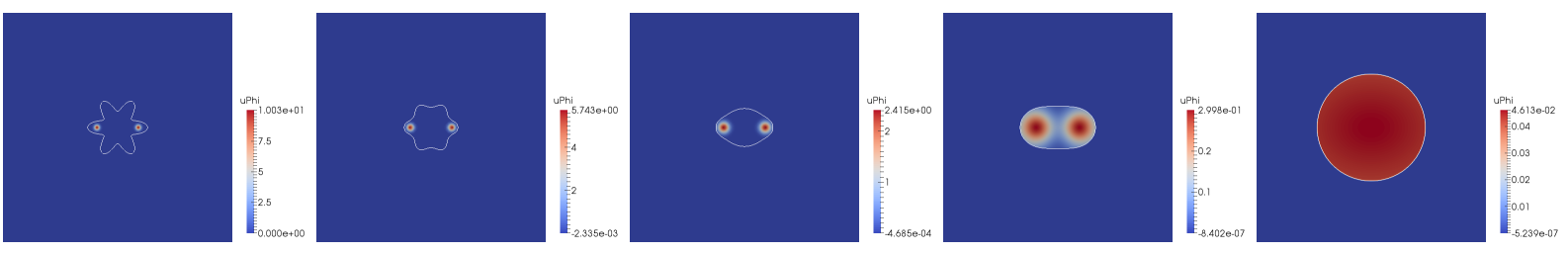

FigURE 7. Discrete concentration $\varphi_{h} u_{h}$ and level set $\left\{\varphi_{h}=\frac{1}{2}\right\}$ at times $t=0$, $t \approx 0.744, t \approx 3.635, t \approx 44.633$ and $t \approx 1501.151$.

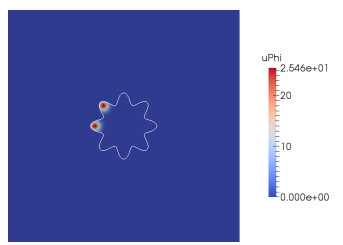

FiguRE 8. Contour plot of cross section of initial concentration $\varphi_{h}(\cdot, 0) u_{h}(\cdot, 0)$ and cross section of level set $\left\{\varphi_{h}(\cdot, 0)=\frac{1}{2}\right\}$.
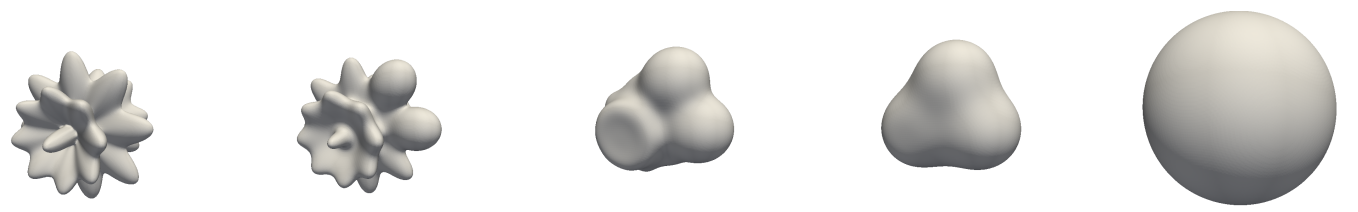

Figure $9 . \quad$ Level sets $\left\{\varphi_{h}(\cdot, t)=\frac{1}{2}\right\}$ for $t=0, t \approx 0.1108, t \approx 0.6041, t \approx 1.176, t \approx 35.8921$.

as an initial condition with $R_{\psi}:=1.7\left(0.5+A_{\psi} \cos \left(k_{\psi} \psi\right)\right)$ and

$$
A_{\theta}=A_{\psi}=0.2 ; \quad k_{\theta}=k_{\psi}=8 .
$$

Further parameters are

$$
D_{+}=0.1 ; \quad \alpha=0.05 ; \quad \beta=1 ; \quad \varepsilon=\frac{1}{8}
$$

and a similar initial condition for $u$ as in the 2d-case, see Fig. 8 for a contour plot of $\varphi_{h}(\cdot, 0) u_{h}(\cdot, 0)$ on a cross section. In Fig. 9 we present level sets $\left\{\varphi_{h}(\cdot, t)=\frac{1}{2}\right\}$ for various times $t$. The locally increased concentration yields a locally deformed membrane shape in the early stage of the evolution, which eventually provides a spherical shape in an almost stationary state.

4.3. Numerical examples for the two-phase model. In this section, we present numerical results for the approximation of the two-phase model. As in the one-phase case 


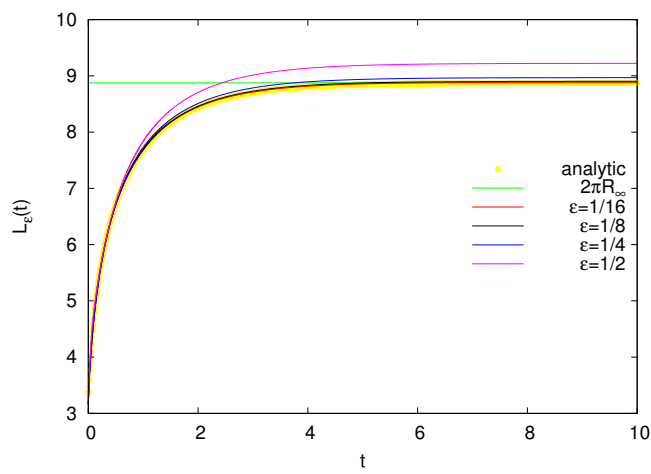

FiguRE 10. Comparison of the analytic perimeter $L=L(t)=2 \pi R(t)$ with $R=R(t)$ given by the ODE 4.15 with initial condition $R_{0}=\frac{1}{2}$ with diffuse length $L_{\varepsilon}$ versus time $t$ for various values of $\varepsilon$.

we validate the numerical scheme by a comparison with analytic solutions. Moreover, we provide results for $n=2,3$ with similar applications as for the one-phase model.

4.3.1. Comparison with analytic results. For the two-phase system (1.5)-(1.8), we obtain in the formal limit $D_{ \pm} \rightarrow \infty$

$$
\begin{aligned}
-\Delta u_{ \pm} & =0 & & \text { in } \Omega_{ \pm}(t), t>0, \\
\nabla u_{ \pm} \cdot \nu & =0 & & \text { on } \Gamma(t), t>0, \\
v & =-\alpha H+\beta[u] & & \text { on } \Gamma(t), t>0, \\
\partial_{n} u_{-} & =0 & & \text { on } \Sigma, t>0 .
\end{aligned}
$$

From 4.11) and (4.12) we deduce, that $u_{ \pm}$are spatially constant $u_{ \pm}(x, t)=\hat{u}_{ \pm}(t)$. Again, $u_{ \pm}$are given by mass conservation

$$
\hat{u}_{ \pm}(t)\left|\Omega_{ \pm}(t)\right|=\int_{\Omega_{ \pm}(t)} u_{ \pm}(x, t) \mathrm{d} x=\int_{\Omega_{ \pm}(0)} u_{ \pm}(x, 0) \mathrm{d} x=\int_{\Omega_{ \pm}(0)} u_{ \pm, 0}(x) \mathrm{d} x,
$$

where constant initial data $u_{ \pm, 0}=u_{ \pm}(\cdot, 0)$ are assumed. Assuming $\Omega=B_{R_{\Sigma}}(0)$ for some $R_{\Sigma}, \Omega_{+}(t)=B_{R(t)}(0) \forall t$ and constant initial data for $u_{ \pm}$, one obtains an ODE

$$
\dot{R}=-\frac{\alpha}{R}+\frac{\beta u_{+, 0} R_{0}^{2}}{R^{2}}-\frac{\beta u_{-, 0}\left(R_{\Sigma}^{2}-R_{0}^{2}\right)}{R_{\Sigma}^{2}-R^{2}}
$$

for the radius $R$, supplemented with an initial datum $R(0)=R_{0}$. Using $R_{0}=0.5, u_{+, 0} \equiv 5$, $u_{-, 0} \equiv 0.5$ and $R_{\Sigma}=5$, we again solve this ODE with Maple ${ }^{\mathrm{TM}}[15]$ and compare the corresponding perimeter with diffuse perimeters $L_{\varepsilon}$ obtained from the discretization of (2.6) - 2.8). For the diffuse-interface approximation we have used a triangulation $\Omega_{h}$ of $\Omega=B_{R_{\Sigma}}(0), \varepsilon=0.1$ and $\delta=10^{-5}$. Furthermore, the parameters $D_{ \pm}=100, \alpha=\frac{1}{8}, \beta=1$ as well as initial conditions $u_{+, 0} \equiv 5$ and $u_{-, 0} \equiv 0.5$ have been applied. In Fig. 10 one can see a comparison of the analytically obtained perimeter in comparison with $L_{\varepsilon}$ for several values of $\varepsilon$. Again, the results of the asymptotic expansions for the limit $\varepsilon \rightarrow 0$ in section 3 are confirmed by the numerical experiments.

Similar to the one-phase case, we further validate the numerical scheme by considering mass conservation

$$
M_{+}(t):=\int_{\Omega} \varphi(x, t) u_{+}(x, t) \mathrm{d} x \equiv M_{+}(0)
$$



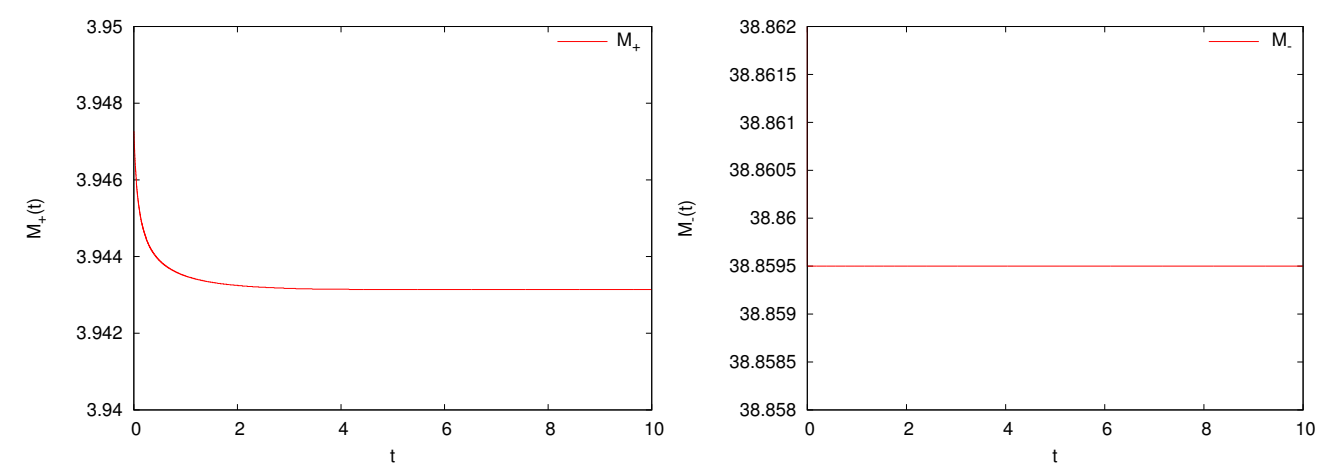

FigURE 11. Discrete counterparts of mass $M_{+}$(left) and $M_{-}(t)$ (right) versus time for $\varepsilon=\frac{1}{8}$.

and

$$
M_{-}(t):=\int_{\Omega}(1-\varphi(x, t)) u_{-}(x, t) \mathrm{d} x \equiv M_{-}(0) .
$$

In Fig. 11 we see the discrete counterparts of mass $M_{+}$and $M_{-}$versus time for the case $\varepsilon=\frac{1}{8}$ of the previous example. The results again further justify the applied numerical treatment.

4.3.2. Further numerical results. For the two-phase model we restrict ourselves to case III, where a perturbation of a circular initial membrane shape and a locally increased initial solute concentration $u_{+, 0}$, while the initial concentration $u_{-, 0}$ is assumed constant. In addition, we present examples with a perturbation of a spherical initial membrane shape in $\mathbb{R}^{3}$.

III: Perturbation of circular/spherical membrane shape and locally increased solute concentration.

2d: We assume

$$
\Omega_{+}(0)=\left\{(r \cos \theta, r \sin \theta): 0 \leq r<R_{\Gamma}+A \cos (m \theta), 0 \leq \theta \leq 2 \pi\right\}
$$

with $A=0.7, m=6, R_{\Gamma}=1.5$ and

$$
\begin{aligned}
u_{0}(x, y)=B\left(a_{u}\right) & {\left[\exp \left(-a_{u}\left(\left(x-x_{0}\right)^{2}+\left(y-y_{0}\right)^{2}\right)\right)\right.} \\
+ & \left.\exp \left(-a_{u}\left(\left(x-x_{1}\right)^{2}+\left(y-y_{1}\right)^{2}\right)\right)\right]
\end{aligned}
$$

for $(x, y) \in \Omega, x_{0}=1.9, y_{0}=0, x_{1}=0.95, y_{1}=1.645448, a_{u}=20$ and $B\left(a_{u}\right)$ is chosen such that $M=\pi$ holds. Moreover, we use $u_{-, 0} \equiv 1$ and the parameters $D_{ \pm}=0.1, \alpha=0.05, \beta=1$. For the diffuse-interface approximation, we choose $\Omega=(-5,5)^{2}, \varepsilon=0.1$ and $\delta=10^{-5}$. In Fig. 12 and Fig. 13 one can see contour plots of the conserved discrete concentrations $\varphi_{h} u_{+, h}$ and $\left(1-\varphi_{h}\right) u_{-, h}$, respectively, as well as the level lines $\left\{\varphi_{h}=\frac{1}{2}\right\}$. The results clearly show an influence of the concentrations on the membrane shape and vice versa. Furthermore, a drift of the membrane to the right can be observed.

3d: For the two-phase system, we consider the same initial membrane shape (4.10) as for the one-phase model. The initial condition for concentration $u_{+}$coincides with the one for $u$ in the one-phase model, as well. In addition, we assume $u_{-}(\cdot, 0)=0.1$, see Fig. 14 contour plots of cross sections of the initial concentrations $\varphi_{h}(\cdot, 0) u_{ \pm, h}(\cdot, 0)$. We further use the parameters 

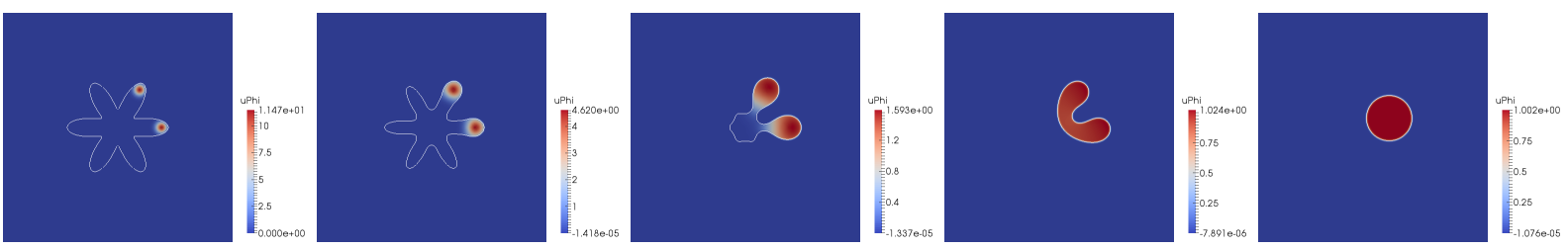

FiguRE 12. Perturbation of a circular initial membrane shape and a locally increased initial solute concentration $u_{+, 0}$, constant initial concentration $u_{-, 0}$. Discrete concentration $\varphi_{h} u_{+, h}$ and level set $\left\{\varphi_{h}=\frac{1}{2}\right\}$ at times $t=0, t \approx 0.191, t \approx 1.427, t \approx 32.328$ and $t \approx 500$.
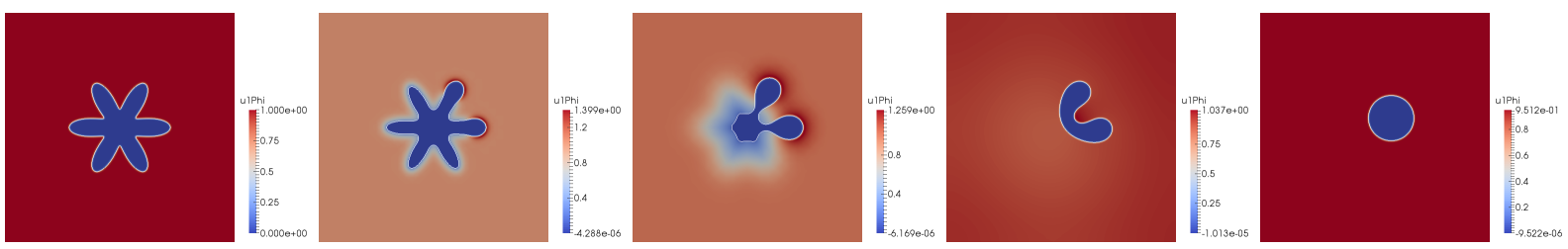

FiguRE 13. Perturbation of a circular initial membrane shape and a locally increased initial solute concentration $u_{+, 0}$, constant initial concentration $u_{-, 0}$. Discrete concentration $\left(1-\varphi_{h}\right) u_{-, h}$ and level set $\left\{\varphi_{h}=\frac{1}{2}\right\}$ at times $t=0, t \approx 0.191, t \approx 1.427, t \approx 32.328$ and $t \approx 500$.
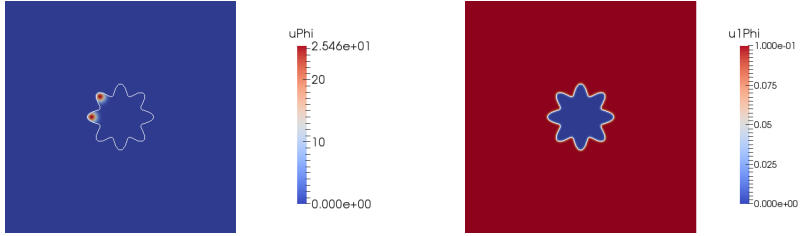

FiguRE 14. Contour plots of cross sections of the initial concentrations $\varphi_{h}(\cdot, 0) u_{ \pm, h}(\cdot, 0)$ and cross section of level set $\left\{\varphi_{h}(\cdot, 0)=\frac{1}{2}\right\}$.
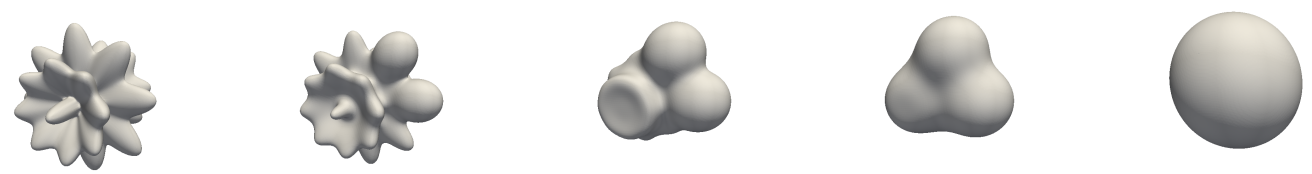

FIGURE 15. Level sets $\left\{\varphi_{h}(\cdot, t)=\frac{1}{2}\right\}$ for $t=0, t \approx 0.1108, t \approx 0.6041, t \approx 1.176, t \approx 35.8921$.

$$
D_{+}=D_{-}=0.1 ; \quad \alpha=0.05 ; \quad \beta=1 ; \quad \varepsilon=\frac{1}{8} ; \quad \delta=10^{-5}
$$

and the domain $\Omega=(-5,5)^{3}$. In Fig. 15 we see a similar evolution as in the case of the one-phase model, which is due to the fact that the concentration $u_{-}$is rather low for this example.

\section{Conclusions}

The "diffuse domain method" has originally been used for the numerical treatment of PDE's and corresponding boundary conditions in domains with possibly complicated boundaries and for the simulation of coupled bulk-surface PDE's [9, 10, 11, 24], see also [27, 16] for the evolving surface case. In this contribution it is used for a new diffuseinterface approximation of a Stefan-like free boundary problem for osmotic swelling, which 
has not been numerically investigated yet. Besides [23], the current paper provides a further example of the flexibility of the diffuse domain method with respect to applicability to more classical free boundary problems.

Acknowledgment. The author would like to thank Georg Prokert and Frieder Lippoth for helpful discussions and especially for pointing out several interesting numerical examples.

\section{REFERENCES}

[1] Abels, H. ; Lam, K. F. ; Stinner, B.: Analysis of the diffuse domain approach for a bulk-surface coupled PDE system. (2015). - arXiv:1502.04902

[2] Alikakos, N.D. ; Bates, P.W. ; Chen, X.: The convergence of solutions of the Cahn-Hilliard equation to the solution of the Hele-Shaw model. In: Arch. Rational Mech. Anal. 128 (1994), Nr. 2, S. $165-205$

[3] Allen, S.M. ; CAhn, J.W.: Microscopic theory for antiphase boundary motion and its application to antiphase domain coarsening. In: Acta Metall. 27 (1979), Nr. 6, S. 1085-1095

[4] Barrett, J.W. ; Nürnberg, R. ; Styles, V.: Finite element approximation of a phase field model for void electromigration. In: SIAM J. Numer. Anal. 42 (2004), Nr. 2, S. 738-772 (electronic)

[5] Burger, M. ; Elvetun, O.L. ; Schlottbom, M.: Analysis of the Diffuse Domain Method for second order elliptic boundary value problems. (2014). - arXiv:1412.5641

[6] Evans, L. C. ; Soner, H. M. ; Souganidis, P. E.: Phase transitions and generalized motion by mean curvature. In: Comm. Pure Appl. Math. 45 (1992), Nr. 9, S. 1097-1123

[7] Frischmuth, K. ; Hänler, M.: Numerical analysis of the closed osmometer problem. In: ZAMM Z. Angew. Math. Mech. 79 (1999), Nr. 2, S. 107-116

[8] Ilmanen, T.: Convergence of the Allen-Cahn equation to Brakke's motion by mean curvature. In: $J$. Differential Geom. 38 (1993), Nr. 2, S. 417-461

[9] Kockelkoren, J. ; Levine, H. ; Rappel, W.-J.: Computational approach for modeling intra- and extracellular dynamics. In: Phys. Rev. E 68 (2003), S. 037702

[10] Levine, H. ; Rappel, W.-J.: Membrane-bound Turing patterns. In: Phys. Rev. E (3) 72 (2005), Nr. 6, S. 061912, 5

[11] Li, X. ; Lowengrub, J. ; Rätz, A. ; Voigt, A.: Solving PDEs in complex geometries: a diffuse domain approach. In: Commun. Math. Sci. 7 (2009), Nr. 1, S. 81-107

[12] Lippoth, F. ; Peletier, M.A. ; Prokert, G.: A moving boundary problem for the Stokes equations involving osmosis: variational modelling and short-time well-posedness. (2014). - arXiv:1409.7252

[13] Lippoth, F. ; Prokert, G.: Classical solutions for a one-phase osmosis model. In: J. Evol. Equ. 12 (2012), Nr. 2, S. 413-434

[14] Lippoth, F. ; Prokert, G.: Stability of equilibria for a two-phase osmosis model. In: NoDEA Nonlinear Differential Equations Appl. 21 (2014), Nr. 1, S. 129-148

[15] MAPLE ${ }^{\mathrm{TM}}$ 16, Maple is a trademark of Waterloo Maple I.:

[16] Marth, W. ; Voigt, A.: Signaling networks and cell motility: a computational approach using a phase field description. In: J. Math. Biol. (2014), Nr. 69, 91-112. http://dx.doi.org/10.1007/ s00285-013-0704-4. - DOI 10.1007/s00285-013-0704-4

[17] ModicA, L.: The Gradient Theory of Phase Transitions and the Minimal Interface Criterion. In: Arch. Rational Mech. Anal. 98 (1987), S. 357-383

[18] Modica, L. ; Mortola, S.: Un esempio di Г-convergenza. In: Boll. Un. Mat. Ital. B (5) 14 (1977), Nr. 1, S. 285-299

[19] Mottoni, P. de ; Schatzman, M.: Development of interfaces in $\mathbf{R}^{N}$. In: Proc. Roy. Soc. Edinburgh Sect. A 116 (1990), Nr. 3-4, S. 207-220

[20] Noorden, T. L. ; EcK, C.: Phase field approximation of a kinetic moving-boundary problem modelling dissolution and precipitation. In: Interfaces Free Bound. 13 (2011), Nr. 1, S. 29-55

[21] Pego, R.: Front migration in the nonlinear Cahn-Hilliard equation. In: Proc. Roy. Soc. London Ser. A 422 (1989), S. 261-278

[22] Pickard, W.F.: Modelling the swelling assay for aquaporin expression. In: J. Math. Biol. 57 (2008), Nr. 6, S. 883-903

[23] Räтz, A.: A new diffuse-interface model for step flow in epitaxial growth. In: IMA J. Appl. Math. (2014). - published online 
[24] Rätz, A. ; RöGER, M.: Symmetry breaking in a bulk-surface reaction-diffusion model for signaling networks. In: Nonlinearity 27 (2014), S. 1805-1827

[25] Redeker, M. ; Pop, I.S. ; Rohde, C: Upscaling of a Tri-Phase Phase-Field Model for Precipitation in Porous Media. In: CASA Report, No. 14-31 (2014)

[26] Rubinstein, L. ; Martuzans, B.: Free boundary problems related to osmotic mass transfer through semipermeable membranes. Gakkōtosho Co., Ltd., Tokyo, 1995 (GAKUTO International Series. Mathematical Sciences and Applications, 4). - vi+205 S.

[27] Teigen, K.E. ; Li, X ; Lowengrub, J. ; Wang, F. ; Voigt, A.: A diffuse-interface approach for modeling transport, diffusion and adsorption/desorption of material quantities on a deformable interface. In: Commun. Math. Sci. 7 (2009), Nr. 4, S. 1009-1037

[28] Vey, S. ; Volgt, A.: AMDiS - Adaptive multidimensional simulations. In: Comput. Visual. Sci. 10 (2007), S. 57-67

[29] ZAAL, M.: Cell swelling by osmosis: a variational approach. In: Interfaces Free Bound. 14 (2012), Nr. 4, S. $487-520$

[30] ZaAl, M.M.: Variational Modeling of Parabolic Free Boundary Problems, VU University of Amsterdam, Diss., 2013. http://hdl.handle.net/1871/40209

[31] ZAAL, M.M.: Well-posedness of a parabolic free boundary problem driven by diffusion and surface tension. In: Math. Method Appl. Sci. 38 (2015), Nr. 2, S. 380-392

E-mail address: andreas.raetz@tu-dortmund.de 


\section{Preprints ab 2012/14}

2015-04 Andreas Rätz

Diffuse-interface approximations of osmosis free boundary problems

2015-03 Margit Rösler and Michael Voit

A multivariate version of the disk convolution

2015-02 Christina Dörlemann, Martin Heida, Ben Schweizer

Transmission conditions for the Helmholtz-equation in perforated domains

2015-01 Frank Klinker

Program of the International Conference

Geometric and Algebraic Methods in Mathematical Physics

March 16-19, 2015, Dortmund

2014-10 Frank Klinker

An explicit description of SL $(2, \mathbb{C})$ in terms of $\mathrm{SO}^{+}(3,1)$ and vice versa

2014-09 Margit Rösler and Michael Voit

Integral representation and sharp asymptotic results for some Heckman-Opdam hypergeometric functions of type $\mathrm{BC}$

2014-08 Martin Heida and Ben Schweizer

Stochastic homogenization of plasticity equations

2014-07 Margit Rösler and Michael Voit

A central limit theorem for random walks on the dual of a compact Grassmannian

2014-06 Frank Klinker

Eleven-dimensional symmetric supergravity backgrounds, their geometric superalgebras, and a common reduction

2014-05 Tomáš Dohnal and Hannes Uecker

Bifurcation of nonlinear Bloch waves from the spectrum in the Gross-Pitaevskii equation

2014-04 Frank Klinker

A family of non-restricted $D=11$ geometric supersymmetries

2014-03 Martin Heida and Ben Schweizer

Non-periodic homogenization of infinitesimal strain plasticity equations

2014-02 Ben Schweizer

The low frequency spectrum of small Helmholtz resonators

2014-01 Tomáš Dohnal, Agnes Lamacz, Ben Schweizer

Dispersive homogenized models and coefficient formulas for waves in general periodic media

2013-16 Karl Friedrich Siburg

Almost opposite regression dependence in bivariate distributions

2013-15 Christian Palmes and Jeannette H. C. Woerner

The Gumbel test and jumps in the volatility process

2013-14 Karl Friedrich Siburg, Katharina Stehling, Pavel A. Stoimenov, Jeannette H. C. Wörner

An order for asymmetry in copulas, and implications for risk management 
Product formulas for a two-parameter family of Heckman-Opdam hypergeometric functions of type BC

2013-12 Ben Schweizer and Marco Veneroni

Homogenization of plasticity equations with two-scale convergence methods

2013-11 Sven Glaser

A law of large numbers for the power variation of fractional Lévy processes

2013-10 Christian Palmes and Jeannette H. C. Woerner

The Gumbel test for jumps in stochastic volatility models

2013-09 Agnes Lamacz, Stefan Neukamm and Felix Otto

Moment bounds for the corrector in stochastic homogenization of a percolation model

2013-08 Frank Klinker

Connections on Cahen-Wallach spaces

2013-07 Andreas Rätz and Matthias Röger

Symmetry breaking in a bulk-surface reaction-diffusion model for signaling networks

2013-06 Gilles Francfort and Ben Schweizer

A doubly non-linear system in small-strain visco-plasticity

2013-05 Tomáš Dohnal

Traveling solitary waves in the periodic nonlinear Schrödinger equation with finite band potentials

2013-04 Karl Friedrich Siburg, Pavel Stoimenov and Gregor N. F. Weiß

Forecasting portfolio-value-at-risk with nonparametric lower tail dependence estimates

2013-03 Martin Heida

On thermodynamics of fluid interfaces

2013-02 Martin Heida

Existence of solutions for two types of generalized versions of the Cahn-Hilliard equation

2013-01 Tomáš Dohnal, Agnes Lamacz, Ben Schweizer

Dispersive effective equations for waves in heterogeneous media on large time scales

2012-19 Martin Heida

On gradient flows of nonconvex functional in Hilbert spaces with Riemannian metric and application to Cahn-Hilliard equations

2012-18 Robert V. Kohn, Jianfeng Lu, Ben Schweizer and Michael I. Weinstein

A variational perspective on cloaking by anomalous localized resonance

2012-17 Margit Rösler and Michael Voit

Olshanski spherical functions for infinite dimensional motion groups of fixed rank

2012-16 Selim Esedoğlu, Andreas Rätz, Matthias Röger

Colliding Interfaces in Old and New Diffuse-interface Approximations of Willmore-flow

2012-15 Patrick Henning, Mario Ohlberger and Ben Schweizer

An adaptive multiscale finite elment method

2012-14 Andreas Knauf, Frank Schulz, Karl Friedrich Siburg

Positive topological entropy for multi-bump magnetic fields 